\title{
Oxidative stress in lung epithelial cells from patients with idiopathic interstitial pneumonias
}

\author{
K. Kuwano*, N. Nakashima*, I. Inoshima*, N. Hagimoto*, M. Fujita*, M. Yoshimi*, T. Maeyama*, \\ N. Hamada*, K. Watanabe", N. Hara*
}

Oxidative stress in lung epithelial cells from patients with idiopathic interstitial pneumonias. K. Kuwano, N. Nakashima, I. Inoshima, N. Hagimoto, M. Fujita, M. Yoshimi, T. Maeyama, N. Hamada, K. Watanabe, N. Hara. (C) ERS Journals Ltd 2003.

ABSTRACT: Lung epithelial cells are a primary target for reactive oxygen species (ROS). ROS can cause oxidative deoxyribonucleic acid modification, such as 8-hydroxy-deoxyguanosine (8-OHdG). A human homologue of the MutT protein (hMTH1) prevents this modification. Mitochondria are the most important cellular source of ROS and may be susceptible to oxidative damage. The purpose of this study is to investigate oxidative stress and mitochondrial damage in lung epithelial cells from idiopathic interstitial pneumonias (IIPs).

The authors analysed 8-OHdG, hMTH1, and mitochondrial proteins on lung specimens from 13 patients with IIPs consisted of eight patients with usual interstitial pneumonia and five patients with nonspecific interstitial pneumonia using Western blot analysis and immunohistochemistry.

Immunoreactivity for 8-OHdG and hMTH1 was significantly increased in the lung epithelial cells from patients with IIPs compared with controls. The expression of hMTH1 was localised in the nuclear and cytoplasmic, but not the mitochondrial, fraction of lung homogenates. Immunoreactivity for mitochondrial protein and cytochrome c oxidase complex subunit IV was increased in the lung epithelial cells from patients with IIPs compared with controls.

The current study concludes that oxidative stress may participate in epithelial cell damage in idiopathic interstitial pneumonia, and that increased mitochondrial mass may associate with increased reactive oxygen species production in idiopathic interstitial pneumonia.

Eur Respir J 2003; 21: 232-240.

\begin{abstract}
* Research Institute for Diseases of the Chest, Graduate School of Medical Sciences, Kyushu University, and ${ }^{\#}$ Fourth Department of Internal Medicine, Fukuoka University, Fukuoka, Japan.
\end{abstract}

Correspondence: K. Kuwano, Research Institute for Diseases of the Chest, Graduate School of Medical Sciences, Kyushu University, 3-1-1, Maidashi, Higashi-ku, Fukuoka 812-8582, Japan. Fax: 81926425389

E-mail: kkuwano@kokyu.med.kyushuu.ac.jp

Keywords: Human homologue of the MutT protein, 8-hydroxy-deoxyguanosine, idiopathic interstitial pneumonias, mitochondria, reactive oxygen species

Received: July 122002

Accepted after revision: September 17 2002

This work was supported by a Grant-inAid for Scientific Research (13670604) from the Ministry of Education, Science and Culture of Japan.
Reactive oxygen species (ROS) can provoke deoxyribonucleic acid (DNA) damage, lipid peroxidation, and activation of various genes whose products are involved in inflammation and cell damage. ROS produce 8-oxo-7, 8-dihydrodeoxyguanosine triphosphate (8-oxo-dGTP), which generates 8-oxo-7, 8-dihydro-2'deoxyguanosine (8-oxo-dG; also known as 8-hydroxydeoxyguanosine, $8-\mathrm{OHdG}$ ) after misincorporation into DNA [1]. Incorporation of $8-\mathrm{OHdG}$ causes a defect in DNA replication, such as base mispairing, random point mutations and deletions [2]. $8-\mathrm{OHdG}$ is known to be a good marker for ROS-mediated DNA modification $[3,4]$.

The Escherichia coli, MutT protein (8-oxo-dGTPase), hydrolyses 8-oxo-dGTP to the monophosphate form, and prevents the misincorporation of nucleotides into DNA and messenger ribonucleic acid (mRNA) during replication and transcription [5]. The human protein, whose enzymatic activities and amino acid sequences are similar to those of MutT, was named human MutT homologue 1 (hMTH1) [6]. It has been reported that hMTH1 gene expression is a molecular marker of oxidative stress [7]. The level of hMTH1 protein was found to increase in mitochondria isolated from postmyocardial infarction hearts, as oxidative stress increased [8].

Lung epithelial cells are always exposed to a variety of stresses, and are a primary target for ROS. High intracellular and extracellular levels of antioxidants protect lung epithelial cells. The generation of ROS is increased in conditions such as inflammation, or exposure to air pollutants and cigarette smoke. ROS and their reactions with lung epithelial cells participate in the pathophysiology of several lung diseases, including pulmonary fibrosis, acute respiratory distress syndrome, and lung cancer [9, 10]. There have been a number of studies demonstrating the increased oxidative stress in idiopathic interstitial pneumonias (IIPs). The spontaneous production of oxidants by lung inflammatory cells and the myeloperoxidase concentration are both increased in the alveolar epithelial lining fluid of patients with IIPs [11]. Nitrotyrosine, a byproduct of protein nitration by peroxynitrite, is increased in lungs of patients with IIP [12]. 
F2-isoprostanes, products of the radical-catalysed lipid peroxidation, are increased in bronchoalveolar lavage fluid (BALF) from patients with interstitial lung diseases [13]. Products of lipid peroxidation, measured as thiobarbituric acid malondialdehyde adducts, in plasma and BALF are increased in IIP [14]. Levels of the 9,11-diene conjugate of linoleic acid and 9, 12linoleic acid expressed as a per cent molar ratio, a measure of free-radical-mediated lipid peroxidation, are elevated in patients with IIP compared with control subjects, and also appear to correlate with clinical disease activity [15]. In contrast, there is a marked reduction in antioxidant capacity, measured as Trolox equivalent antioxidant capacity, in the plasma and BALF from patients with IIP [14]. These results demonstrate the evidence of increased oxidative stress and of oxidant/antioxidant imbalance in patients with IIP. However, oxidative DNA damage in lung epithelial cells from patients with IIPs has not been assessed. Therefore, the immunoreactivity for $8-\mathrm{OHdG}$ and hMTH1 expression as indicators of oxidative stress in lung epithelial cells was examined by immunohistochemistry and Western blot analysis.

Mitochondria consume $\sim 90 \%$ of the oxygen used by the body, and $1-2 \%$ of the oxygen metabolised by the mitochondria is converted to ROS [16]. Therefore, mitochondria are the most important cellular source of ROS and may be susceptible to oxidative damage. Impaired mitochondrial function may lead to impaired electron transport and enhanced production of ROS. Increased mitochondrial mass may also lead to the increased production of ROS. Therefore, the mitochondrial mass and the expression of the mitochondriaspecific protein, cytochrome c oxidase complex IV (COX complex IV) was examined in order to assess mitochondrial damage.

\section{Materials and methods}

\section{Materials}

This study of IIPs was performed on 13 lung samples obtained by thoracoscopic lung biopsy. The diagnosis of IIPs was established by a combination of medical history, physical examination, laboratory tests, chest roentgenograms, pulmonary function tests, and the results of histological findings, according to previously described criteria [17]. The histological findings in lung biopsy specimens were compatible with those of usual interstitial pneumonia (UIP) in eight patients and nonspecific interstitial pneumonia (NSIP) in five patients. The characteristics of the patients with IIPs are shown in table 1 . Three observers without knowledge of clinical, physiological, or pathological data reviewed computed tomography (CT) scans independently. Observers scored three CT images with $2 \mathrm{~mm}$ thickness taken at the level of the aortic arch, the carina, and $1 \mathrm{~cm}$ above the diaphragm. Each image was scored on a scale of $0-5$ for both alveolar and interstitial abnormality: alveolar score 0 , no ground glass opacity; $1,<5 \% ; 2,5-25 \% ; 3,25-49 \% ; 4,50$ $75 \%$; 5, >75\%; interstitial score 0 , no fibrosis; 1 , interlobular septal thickening, no discrete honeycombing; 2, honeycombing $<25 \%, 3,25-49 \%$; 4 , $50-75 \%$; 5, >75\%, according to the scoring by KaZerooni et al. [18]. The composite mean of three observers was calculated as the CT score of each patient. The results regarding IIPs were compared with those in eight normal lung parenchyma specimens obtained by lobectomy for lung cancer of solitary pulmonary nodules. These patients comprised five males and three females, whose ages ranged from 56-78 yrs (mean $67 \mathrm{yrs}$ ), and all were smokers. After

Table 1.-Pulmonary function data and computed tomography (CT) score of the subjects with idiopathic interstitial pneumonias

\begin{tabular}{|c|c|c|c|c|c|c|c|c|c|}
\hline Patient & Sex & Age yrs & Brinkman Index & $\% \mathrm{VC}$ & $\% D \mathrm{~L}, \mathrm{CO}$ & $P \mathrm{a}, \mathrm{O}_{2}$ & CT int & CT alv & CT total \\
\hline \multicolumn{10}{|l|}{ UIP } \\
\hline 1 & M & 66 & 1800 & 63 & 56 & 79 & 2.48 & 0.48 & 2.96 \\
\hline 2 & M & 63 & 920 & 103 & 53 & 84 & 1.63 & 0.14 & 1.67 \\
\hline 3 & $\mathrm{M}$ & 52 & 0 & 62 & 67 & 81 & 1.67 & 0.78 & 2.45 \\
\hline 4 & M & 67 & 1250 & 69 & 71 & 84 & 1.96 & 0.22 & 2.18 \\
\hline 5 & M & 55 & 150 & 76 & 75 & 83 & 1.96 & 0.37 & 2.33 \\
\hline 6 & M & 65 & 800 & 101 & 71 & 88 & 1.52 & 0.44 & 1.96 \\
\hline 7 & M & 59 & 800 & 84 & 84 & 73 & 1.74 & 0.74 & 2.48 \\
\hline 8 & M & 42 & 500 & 46 & 68 & 78 & 1.81 & 0.68 & 2.49 \\
\hline Mean & & 58.6 & 777 & 76 & 68 & 81 & 1.85 & 0.47 & 2.32 \\
\hline SD & & 8.6 & 581 & 20 & 10 & 4.6 & 0.30 & 0.26 & 0.39 \\
\hline \multicolumn{10}{|l|}{ NSIP } \\
\hline 1 & $\mathrm{~F}$ & 53 & 0 & 57 & 63 & 65 & 0.22 & 2.30 & 2.52 \\
\hline 2 & $\mathrm{~F}$ & 56 & 0 & 55 & 60 & 75 & 0.41 & 1.48 & 1.89 \\
\hline 3 & M & 68 & 1500 & 93 & 46 & 86 & 0.98 & 1.67 & 2.65 \\
\hline 4 & M & 64 & 0 & 94 & 81 & 76 & 0.32 & 1.22 & 1.54 \\
\hline 5 & M & 50 & 0 & 73 & 57 & 70 & 0.18 & 2.27 & 2.45 \\
\hline Mean & & 57.5 & 300 & 74 & 61 & 74 & 0.42 & 1.79 & 2.21 \\
\hline SD & & 7.6 & 670 & 19 & 13 & 7.8 & 0.32 & 0.48 & 0.47 \\
\hline
\end{tabular}

UIP: usual interstitial pneumonia; NSIP: nonspecific interstitial pneumonia; VC: vital capacity; DL,CO: carbon monoxide diffusion capacity of the lung; $P \mathrm{a}_{2} \mathrm{O}_{2}$ : arterial oxygen tension; F: female; M: male; CT int: CT score for interstitial abnormality; CT alv: CT score for alveolar abnormality. 
the lung tissue was obtained, it was immediately frozen in liquid nitrogen and stored at $-80^{\circ} \mathrm{C}$.

The specific antibodies used in this study were as follows: mouse anti-8-OHdG monoclonal antibody (NOF Corporation, Tokyo, Japan), rabbit anti-hMTH1 polyclonal antibody (NOVUS Biologicals, Littleton, CO, USA), mouse antimitochondrial protein antibody (MAB1273; Chemicon International, Temecula, CA, USA), and mouse antibovine cytochrome oxidase subunit IV monoclonal antibody (20E8-C12; Molecular Probes, Eugene, OR, USA). The same antibodies were used for Western blot and for immunohistochemistry.

\section{Western blot analysis}

Frozen lung tissues were homogenised on ice for $30 \mathrm{~s}$ (6-8 strokes) with a Potter-Elvehjem homogeniser in buffer $\mathrm{A}(25 \mathrm{mM}$ Hepes, $\mathrm{pH} 7.5,5 \mathrm{mM} \mathrm{MgCl}, 1 \mathrm{mM}$ egtazic acid (EGTA), $1 \mathrm{mM}$ phenylmethyl sulfonyl fluoride (PMSF), $1 \mu \mathrm{g} \cdot \mathrm{mL}^{-1}$ leupeptin and $1 \mu \mathrm{g} \cdot \mathrm{mL}^{-1}$ aprotinin). After incubation on ice for $10 \mathrm{~min}$, extracts were vortexed for $10 \mathrm{~s}$ and centrifuged at $800 \times g$ for 2 min at $4{ }^{\circ} \mathrm{C}$ to pellet the nuclei. The supernatant was centrifuged at $15,000 \times g$ for $30 \mathrm{~min}$ at $4^{\circ} \mathrm{C}$. The cleared supernatant was taken as the cytoplasmic extract and the pellet as the mitochondria-rich fraction. The cytoplasmic extract was diluted with $3 \times$ sodium dodecylsulphate (SDS)-sample buffer and boiled. The mitochondria-enriched fraction was directly dissolved in SDS-sample buffer and boiled. Nuclear pellets were incubated in $50 \mu$ buffer B $(25 \mathrm{mM}$ Hepes, $\mathrm{pH} 7.5$, $420 \mathrm{mM} \mathrm{NaCl}, 5 \mathrm{mM} \mathrm{MgCl}, 1 \mathrm{mM}$ EGTA, $1 \mathrm{mM}$ PMSF, $1 \mu \mathrm{g} \cdot \mathrm{mL}^{-1}$ leupeptin, $1 \mu \mathrm{g} \cdot \mathrm{mL}^{-1}$ aprotinin and $25 \%$ glycerol) on ice for $30 \mathrm{~min}$ and then centrifuged at $15,000 \times g$ for $30 \mathrm{~min}$ at $4{ }^{\circ} \mathrm{C}$. The supernatant was taken as the nuclear extract. The cytoplasmic and nuclear extract was diluted with $3 \times$ SDS-sample buffer and boiled. Protein concentrations were determined using the Bio-Rad protein assay. Thirty micrograms of protein per lane were separated by SDS-polyacrylamide gel electrophoresis (SDS-PAGE). After SDS-PAGE, the proteins were transferred to a polyvinylidene fluoride hydrophobic membrane (Millipore, Bedford, MA, USA). Membranes were blocked by $5 \%$ nonfat dry milk in Tris buffer saline containing $0.05 \%$ Tween-20 (TBST) at $4{ }^{\circ} \mathrm{C}$ for $2 \mathrm{~h}$. The membranes were rinsed with TBST and incubated with specific antibodies in blocking buffer at $4{ }^{\circ} \mathrm{C}$ overnight. After being rinsed, the membranes were incubated with biotinylated secondary antibodies for $30 \mathrm{~min}$ at room temperature. The blots were developed using an ECL western blotting detection kit (Amersham Pharmacia Biotech, Buckinghamshire, UK). Pictures of the membranes were taken, scanned and analysed.

\section{Immunohistochemistry}

The tissue samples were fixed in $10 \%$ formalin overnight, and embedded in paraffin. A 5- $\mu \mathrm{m}$ paraffin section was adhered to slides pretreated with poly-Llysine. These sections were dewaxed by washing three times for $5 \mathrm{~min}$ each in xylene, then dehydrated in
$100 \%, 95 \%$ and $80 \%$ ethanol, for $5 \mathrm{~min}$ each, before finally being rinsed with distilled water. For $8-\mathrm{OHdG}$ immunostaining, tissue sections were pretreated with $100 \mu \mathrm{g} \cdot \mathrm{mL}^{-1}$ ribonuclease $\mathrm{A}$ in phosphate buffer saline (PBS) in order to inhibit nonspecific binding to RNA. Immunohistochemistry was performed using a modified streptavidin-biotinylated peroxidase technique using a Histofine SAB-PO kit from Nichirei Corporation (Tokyo, Japan). Nonspecific protein staining was blocked by rabbit or goat serum for $30 \mathrm{~min}$ at room temperature. The sections were incubated with primary antibody at $4^{\circ} \mathrm{C}$ overnight. The sections were rinsed with PBS and incubated with biotinylated secondary antibodies for $30 \mathrm{~min}$. They were then washed and treated with $0.3 \%$ hydrogen peroxide in methanol for $30 \mathrm{~min}$ in order to inhibit the activity of any endogenous peroxide. The slides were washed, incubated with streptavidin-biotin-peroxidase complex for $30 \mathrm{~min}$, and developed according to the manufacturer's directions. The sections were subsequently counterstained with haematoxylin and mounted. The degree of staining was graded from $0-3$ according to the percentage of immunoreactive cells among the bronchiolar and alveolar epithelial cells $(0,0 \% ; 1$, $<25 \% ; 2,25-50 \% ; 3,>50 \%$ ) per field, with $100 \times$ magnification. The fibrosis in each field was also assessed, using the criteria of AsHCROFT et al. [19] with slight modifications, and graded from $0-5$ (0, normal lung; 1, minimal fibrous thickening of alveolar or bronchiolar walls; 2, moderate thickening of walls without obvious damage to lung architecture; 3, increased fibrosis with definite damage to lung structure and formation of fibrous bands or small fibrous masses; 4, severe distortion of lung structure and large fibrous areas ("honeycomb lung" is placed in this category); 5, total fibrous obliteration throughout the field). The immunoreactivity grade and fibrotic grade per field were determined at the same time by two observers. The two observers agreed with each other regarding both grades. The mean grade of immunoreactivity per field was calculated for each patient. Twenty to 50 fields per case were assessed.

\section{Statistics}

The optical density of Western blot analysis was analysed by analysis of variance followed by Scheffe's $\mathrm{F}$ test. The difference in immunostaining grade was analysed by the Kruskal-Wallis test followed by the Mann-Whitney U-test. The correlation between immunostaining grade and pulmonary function tests were analysed by Spearman's rank correlation. A p-value $<0.05$ was considered statistically significant.

\section{Results}

8-oxo-7, 8-dihydro-2'-deoxyguanosine immunohistochemistry

Immunohistochemistry. Positive signals for $8-\mathrm{OHdG}$ were predominantly detected in the nucleus of bronchiolar and alveolar epithelial cells from patients with 

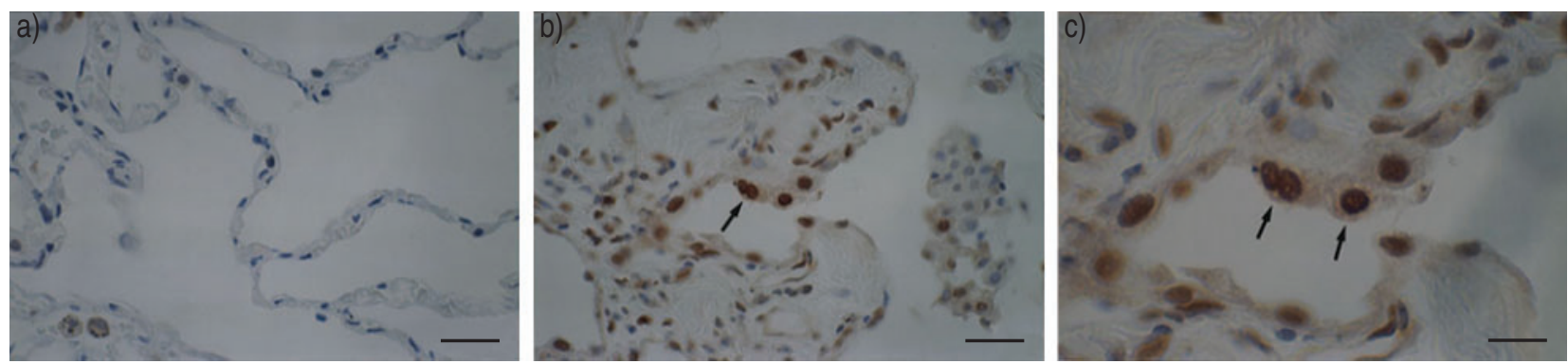

Fig. 1.-Representative results of immunohistochemical analysis for 8-hydroxy-deoxyguanosine in a) normal lung parenchyma and b, c) lung tissues from patients with idiopathic interstitial pneumonias. Positive signals were predominantly detected in the nuclei of epithelial cells (arrows). Scale bars: $\mathrm{a}$ and $\mathrm{b}=20 \mu \mathrm{m} ; \mathrm{c}=10 \mu \mathrm{m}$.

IIPs, but less positive signals were detected in the controls (fig. 1). Semiquantitative results of the immunostaining grade in lung epithelial cells show that there was a significant increase in UIP and NSIP, compared with the controls (fig. 2a). In both UIP and NSIP, positive signals were significantly increased in lesions
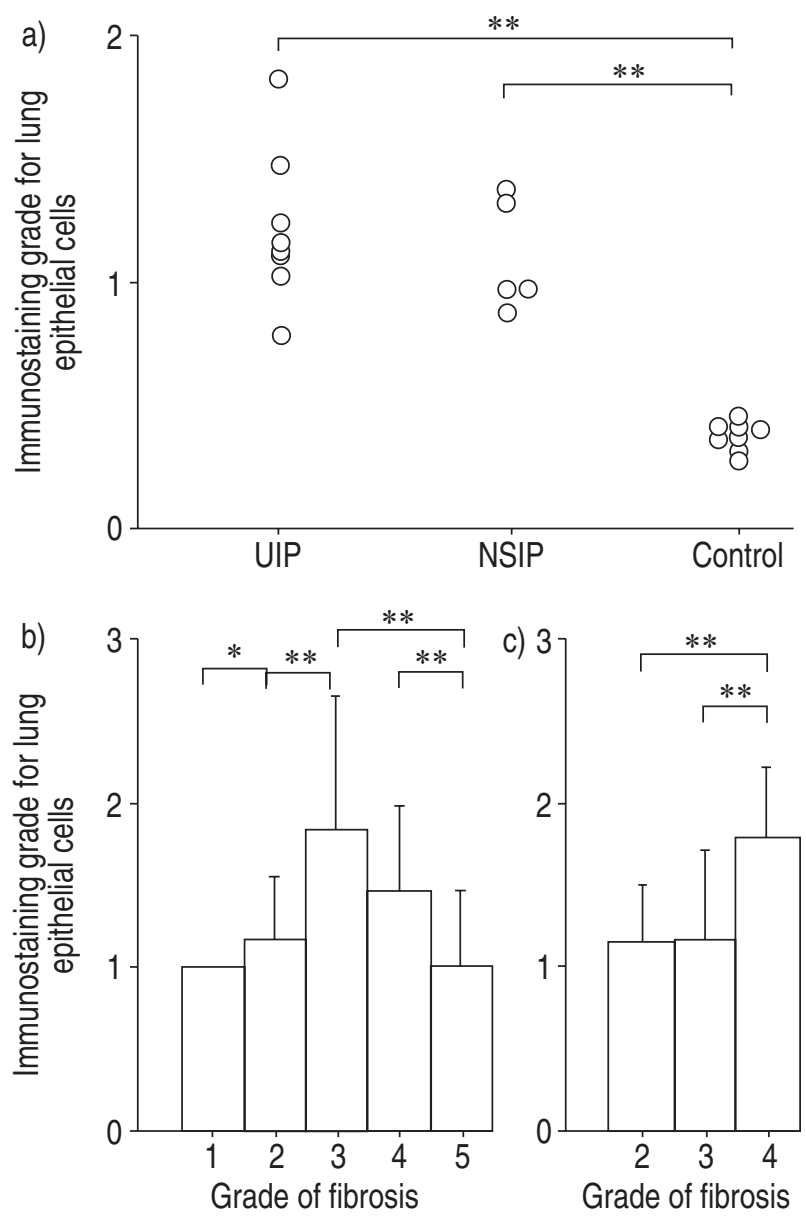

Fig. 2.-a) Summary of immunostaining grade for 8-hydroxydeoxyguanosine (8-OHdG) in lung epithelial cells from usual interstitial pneumonia (UIP) $(n=8)$, nonspecific interstitial pneumonia (NSIP) $(n=5)$ and control $(n=8)$ subjects. Each circle represents one individual. Association between immunostaining grade for $8-\mathrm{OHdG}$ in lung epithelial cells and the grade of pulmonary fibrosis in each field under light microscopy in b) UIP and c) NSIP. *: $\mathrm{p}<0.05 ; * *: \mathrm{p}<0.01$ showing a moderate grade of fibrosis (fig. 2b). Immunostaining grade for $8-\mathrm{OHdG}$ did not correlate with pulmonary function tests or CT scores.

\section{Immunoreactivity of the human homologue of the MutT protein}

Western blot analysis. The representative results of Western blot analysis for hMTH1 show that the expression was detected in the cytoplasm and nucleus, but not in the mitochondrial fraction (fig. 3a). The quantitative results of Western blot analysis show that the expression in the cytoplasm and nucleus was variable but increased in IIPs compared with the controls (fig. 3b).

Immunohistochemistry. Positive signals for hMTH1 were predominantly detected in the nucleus and
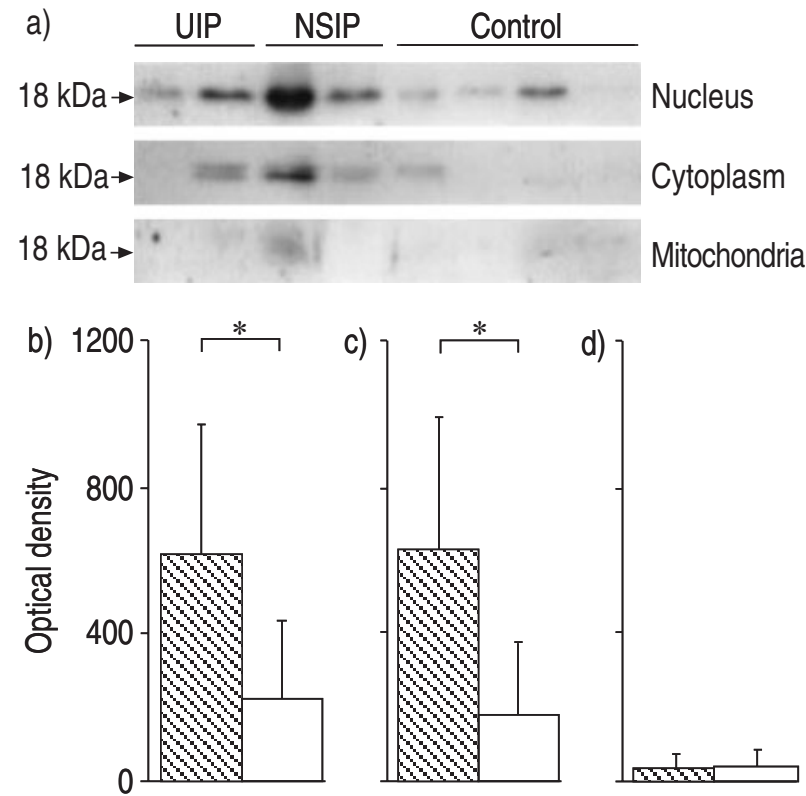

Fig. 3.-a) Representative results of Western blot analysis, and quantitative results of the analysis, for the human homologue of the MutT protein in the b) nuclear, c) cytoplasmic, and d) mitochrondria-rich fractions in lung tissues from patients with usual interstitial pneumonia (UIP), nonspecific interstitial pneumonia (NSIP) (n=13 for UIP+NSIP; $\mathbb{\mathbb { N }}$ ) and normal lung parenchyma $(\mathrm{n}=8 ; \square) .{ }^{*}: \mathrm{p}<0.05$. 

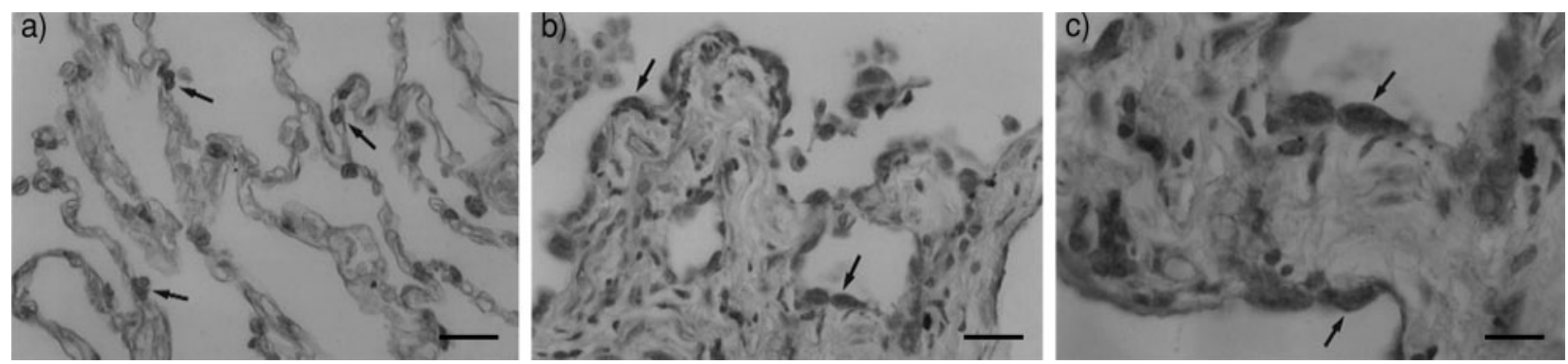

Fig. 4.-Representative results of immunohistochemical analysis for the human homologue of the MutT protein in a) normal lung parenchyma and $b, c)$ lung tissues from patients with idiopathic interstitial pneumonias. Positive signals were predominantly detected in the nuclei and cytoplasm of epithelial cells (arrows). Scale bars: $a$ and $b=20 \mu \mathrm{m} ; \mathrm{c}=10 \mu \mathrm{m}$.

cytoplasm of bronchiolar and alveolar epithelial cells from patients with UIP and NSIP, and from the controls (fig. 4). Semiquantitative results of immunostaining grade in lung epithelial cells show that there was a significant increase in UIP and NSIP compared with the controls (fig. 5a). Positive signals were significantly
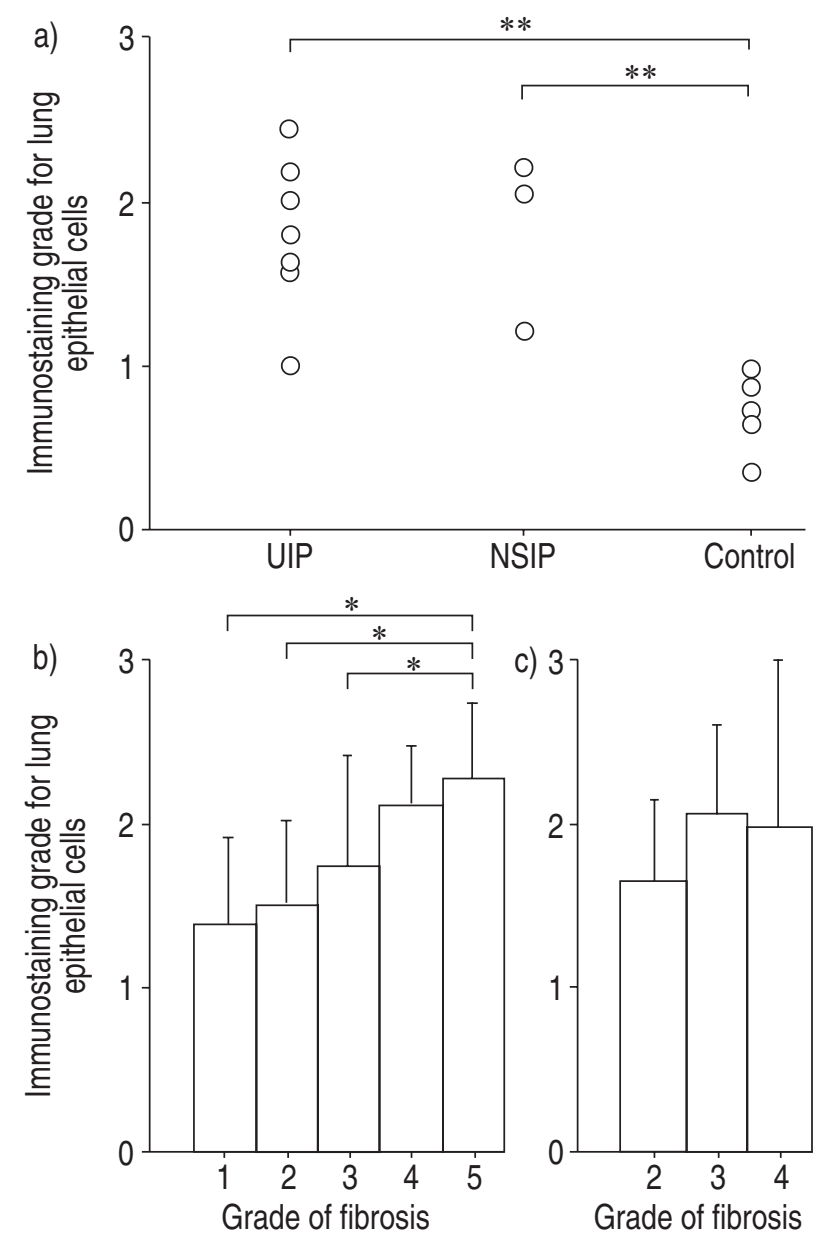

Fig. 5.-a) Summary of immunostaining grade for the human homologue of the MutT protein (hMTH1) in lung epithelial cells from interstitial pneumonia (UIP) $(n=8)$, nonspecific interstitial pneumonia (NSIP) $(n=5)$ and control $(n=8)$ subjects. Each circle represents one individual. Association between immunostaining grade for hMTH1 in lung epithelial cells and the grade of pulmonary fibrosis in each field under light microscopy in b) UIP and c) NSIP. *: $\mathrm{p}<0.05 ; * *: \mathrm{p}<0.01$. increased, accompanied by the progression of fibrosis in UIP (fig. 5b). Immunostaining grade for hMTH1 did not correlate with pulmonary function tests or CT scores.

\section{Expression of mitochondrial protein}

Immunohistochemistry. To examine whether mitochondrial mass is changed in lung epithelial cells from patients with IIPs, mitochondrial protein expression was analysed. Although antimitochondrial protein antibody reacts with an uncharacterised protein, the immunoreactivity shows a beaded or granular pattern that is typical of mitochondria, as described previously [20]. Positive signals for mitochondrial protein were predominantly detected in lung epithelial cells and alveolar macrophages from patients with IIPs, and from the controls (fig. 6). The immunostaining grade for mitochondrial protein in lung epithelial cells was significantly increased in both UIP and NSIP compared with the controls (fig. 7a). Strong signals were predominantly detected in hyperplastic epithelial cells. The degree of positive staining for mitochondrial protein in lung epithelial cells was increased accompanied by the progression of fibrosis in both UIP and NSIP (fig. 7b).

\section{Expression of cytochrome c oxidase complex IV}

Western blot analysis. The representative results of Western blot analysis for COX complex IV show that the expression was detected in the mitochondrial fraction specifically (fig. 8a). The quantitative results of Western blot analysis show that there was a significant increase in COX complex IV content in the same amount of proteins from the mitochondrial fraction in UIP compared with the controls (fig. 8b).

Immunohistochemistry. To examine whether the expression of mitochondria-specific protein is changed in lung epithelial cells from patients with IIPs, COX complex IV expression was analysed. Positive staining was detected in lung epithelial cells from patients with UIP and NSIP, and from the controls. Strong signals were detected in the hyperplastic epithelial cells in UIP and NSIP (fig. 9). 

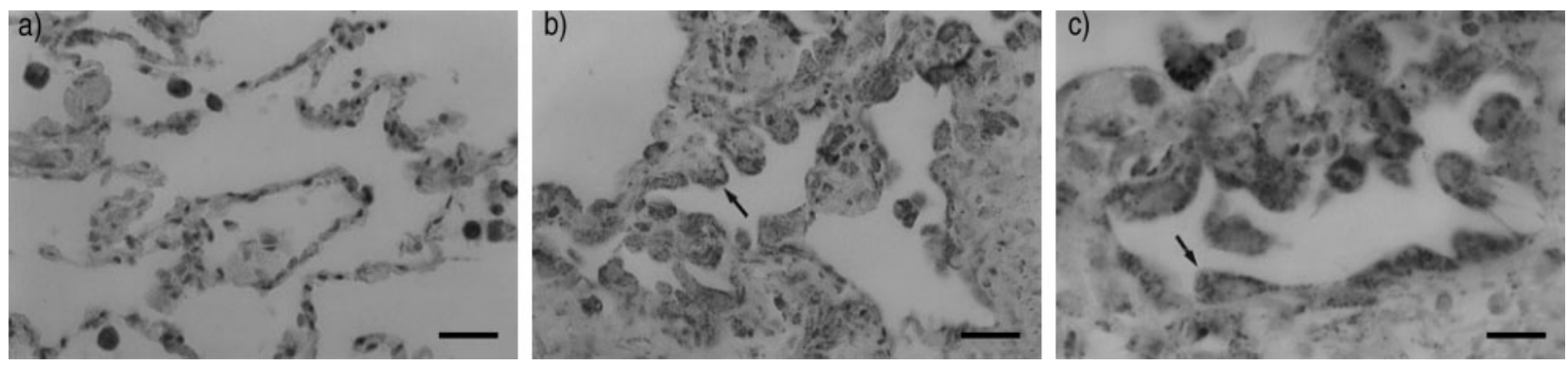

Fig. 6. - Representative results of immunohistochemical analysis for mitochondrial protein in a) normal lung parenchyma and b, c) lung tissues from patients with idiopathic interstitial pneumonias (IIP). Strong positive signals were predominantly detected in the hyperplastic epithelial cells in IIP (arrows). Scale bars: $a$ and $b=20 \mu \mathrm{m} ; \mathrm{c}=10 \mu \mathrm{m}$.

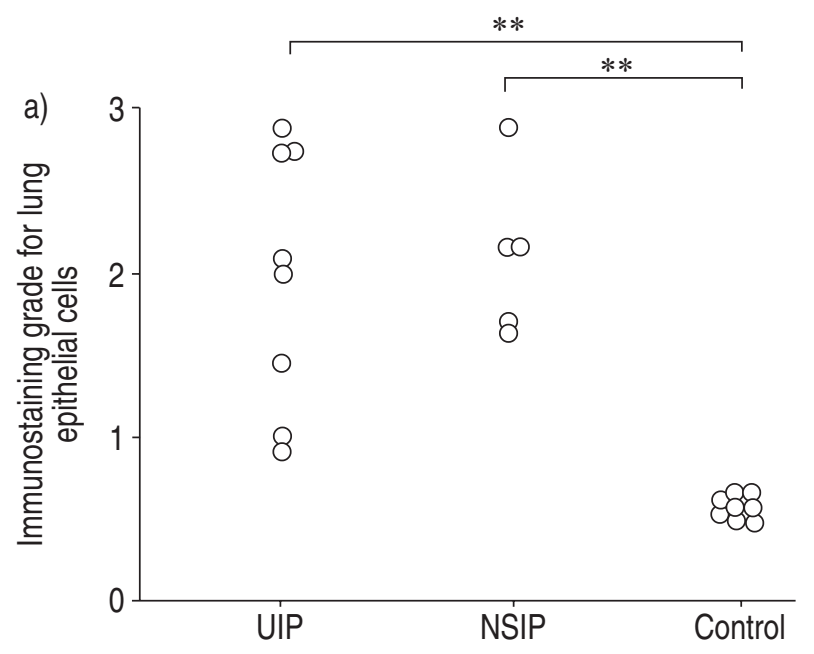

8-OHdG in situ, without the risk of artifactorial production of 8-OHdG, during the extraction and hydrolytic processes [21]. Although positive signals were detectable in lung epithelial cells of the controls, such signals showed a significant increase in epithelial cells from patients with UIP and NSIP. Positive signals for 8-OHdG were predominantly localised in the nuclei, whereas weak staining was found in the cytoplasm in some cells. Positive signals for hMTH1 by immunohistochemistry were also significantly increased in epithelial cells from IIPs, compared with the controls, which was compatible with the results of immunohistochemistry for $8-\mathrm{OHdG}$. The degree of positive staining for hMTH1 was increased, accompanied by the progression of pulmonary fibrosis in UIP and NSIP, whereas that for $8-\mathrm{OHdG}$ was significantly increased in lesions showing a moderate grade of
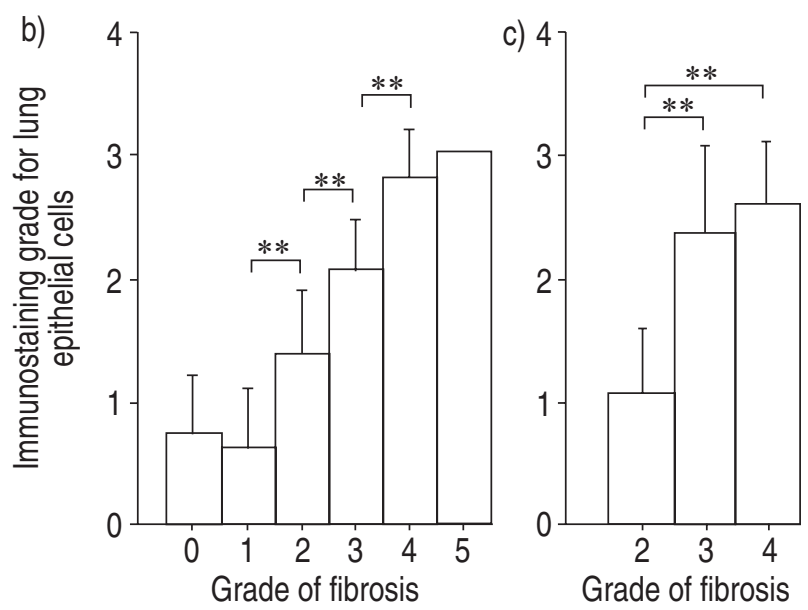

Fig. 7. -a) Summary of immunostaining grade for mitochondrial protein in lung epithelial cells from usual interstitial pneumonia (UIP) $(n=8)$, nonspecific interstitial pneumonia (NSIP) $(n=5)$ and control $(n=8)$ subjects. Each circle represents one individual. Association between immunostaining grade for mitochondrial protein in lung epithelial cells and the grade of pulmonary fibrosis in each field under light microscopy in b) UIP and c) NSIP. **: $\mathrm{p}<0.01$.

\section{Discussion}

Immunohistochemical detection of $8-\mathrm{OHdG}$ has advantages over high-performance liquid chromatography (HPLC) in that it allows the localisation of

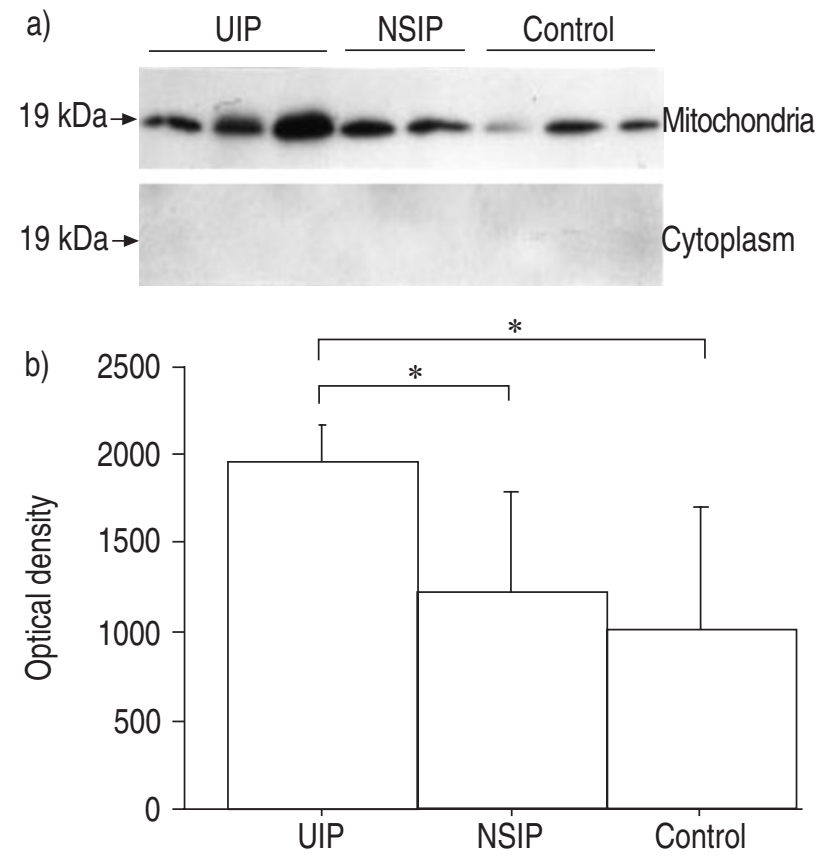

Fig. 8.-a) Representative results of Western blot analysis for cytochrome c oxidase (COX) complex IV in the cytoplasmic and mitochondria-rich fractions in lung tissues from patients with usual interstitial pneumonia (UIP), nonspecific interstitial pneumonia (NSIP) and normal lung parenchyma. b) Quantitative results of Western blot analysis for COX complex IV in the mitochondria-rich fractions. $*$ : $\mathrm{p}<0.05$. 

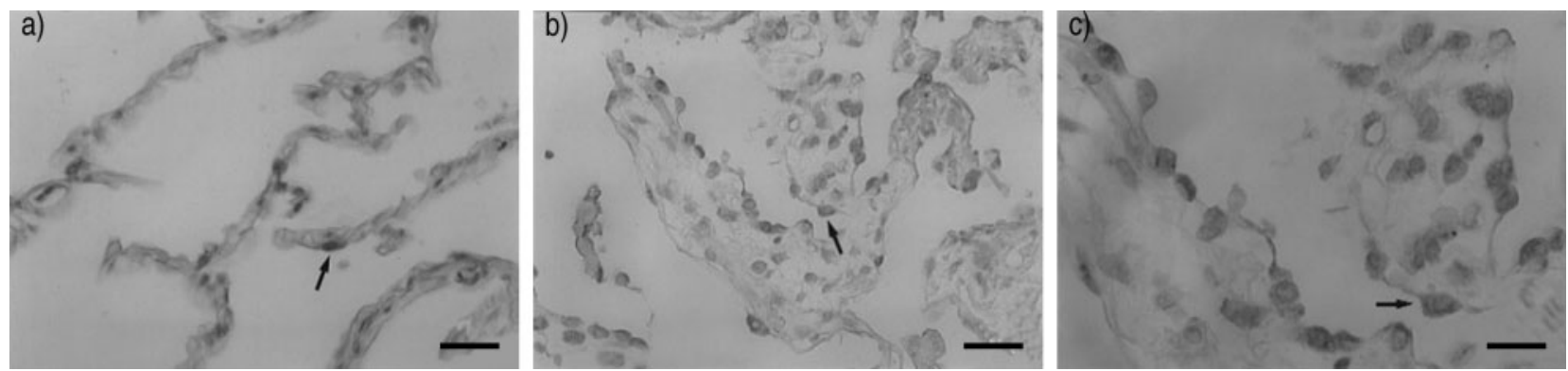

Fig. 9. - Representative results of immunohistochemical analysis for cytochrome c oxidase complex IV in a) normal lung parenchyma and b, c) lung tissues from patients with idiopathic interstitial pneumonias (IIP). Strong positive signals were predominantly detected in the hyperplastic epithelial cells in IIP (arrows). Scale bars: $a$ and $b=20 \mu \mathrm{m} ; \mathrm{c}=10 \mu \mathrm{m}$.

fibrosis. These results suggest that oxidative DNA damage is increased in the lung epithelial cells of patients with IIPs, and that the damage in hyperplastic or regenerating epithelial cells in advanced fibrotic lesions may be partly repaired.

Although 8-OHdG is increased in the smoker's lung compared with the nonsmoker's lung [22], all of the control samples used were obtained from smokers. Therefore, the effect of smoking on the immunoreactivity for $8-\mathrm{OHdG}$ can be ignored. The control lungs were obtained by lobectomy for lung cancer. INOUE et al. [23] demonstrated that lung cancer patients have increased $8-\mathrm{OHdG}$ levels in peripheral lung tissue DNA, and suggested that oxidative stress is induced in entire lung, either by environmental agents or under the influence of genetic factors in patients with lung cancer. In IIPs, inflammatory cells have the potential to damage lung epithelial cells by releasing proteases and ROS, as well as cytokines, chemokines and growth factors, in addition to environmental factors and genetic factors. Therefore, 8-OHdG levels may be increased in lung tissues from patients with IIPs compared with those with lung cancer. Increased levels of $8-\mathrm{OHdG}$ in lung tissues from patients with IIPs compared with lung parenchyma from patients with lung cancer may partly explain the high incidence of lung cancer among patients with IIPs.

Previous studies have shown oxidative stress in lung tissues of IIP using other parameters. Peroxynitrite is a potent oxidant and nitrotyrosine is a by-product of protein nitration by peroxynitrite. SALEH et al. [12] demonstrated a significant increase in the immunoreactivity for nitrotyrosine and inducible nitric oxide synthase in alveolar epithelium at early-to-intermediate stage compared with control subjects or end-stage subjects using immunohistochemistry. These results seem to be compatible with those of $8-\mathrm{OHdG}$, because immunoreactivity for $8-\mathrm{OHdG}$ was significantly increased in lesions showing a moderate grade of fibrosis.

RAHMAN et al. [14] demonstrated that products of lipid peroxidation in BALF were increased in patients with IIP whether they were smokers or nonsmokers. They speculated that smoking does not enhance the process of peroxidation that was already present in patients with IIP. These results may be compatible with the present authors' results that there was a significant increase of immunoreactivity for $8-\mathrm{OHdG}$ in patients with NSIP, who were nonsmokers except one, compared with control lung tissues from patients with lung cancer who were all smokers.

Mitochondria are the most important cellular source of ROS and may be susceptible to oxidative damage. Therefore, the authors had expected that mitochondria might be damaged by oxidative stress. However, positive signals for $8-\mathrm{OHdG}$ were predominantly localised in the nuclei. Positive signals for hMTH1 were localised in the nuclei and cytoplasm by immunohistochemistry, and the results of Western blot analysis demonstrated that hMTH1 protein was expressed in the nuclear and cytoplasmic fractions, but not in the mitochondrial fraction. These results were unexpected and may suggest that oxidative damage is not remarkable in the mitochondria from patients with IIPs.

There was a significant variability of hMTH1 expression in Western blot analysis. A lack of consistency in the measurement of $8-\mathrm{OHdG}$ by HPLC has been reported; 8-OHdG levels in normal human cells ranged from $<0.1->200$ per $10^{6}$ base pairs [24]. Therefore, hMTH1 levels may also be variable in Western blot analysis. Another possibility is that lung homogenates consist not only of epithelial cells but also of other types of cells, such as macrophages and inflammatory cells expressing significant amounts of hMTH1, as demonstrated by immunohistochemistry.

It has recently been reported that 8-oxo-dGTPase was detected in the mitochondrial fractions isolated from failing hearts following myocardial infarction in mice, using Western blot analysis with an antibody to its human homologue, hMTH1 [8]. Furthermore, it was also reported that the immunoreactivity for $8-\mathrm{OHdG}$ was found to a remarkable degree in the cytoplasm of brain cells from patients with Alzheimer's disease. The immunoreactivity for mitochondrial protein and COX complex IV was decreased in brain cells from patients with Alzheimer's disease compared with normal brain cells [20].

In this study, the immunoreactivity for mitochondrial protein and COX complex IV protein was significantly increased in lung epithelial cells, especially hyperplastic epithelial cells from patients with IIPs, compared with the controls, by immunohistochemistry. The results of immunoreactivity for COX complex IV in the mitochondrial fraction by Western blot analysis seem to suggest the preservation of mitochondrial function. These differences from previous reports may be due to 
cell or tissue specificity. Since the lung epithelium is constantly exposed to ROS, the lung epithelium is enriched with antioxidants [25]. Other scavengers or repair enzymes may abrogate oxidative stress on the mitochondria in lung epithelial cells, otherwise mitochondrial damage may lead to impaired electron transport, enhanced production of ROS, and further damage to epithelial cells.

This study demonstrates increased oxidative stress in the lung epithelial cells from patients with IIPs compared with normal lung parenchyma, although the number of patients was small. Oxidative stress in many pathological conditions has been linked to apoptosis. ROS are shown to upregulate the Fas-Fas ligand pathway and the release of cytochrome c [26, 27], and also to activate p53 [28]. The activation of p53 leads to the upregulation of Bax [29] and Fas [30]. In fact, DNA damage and apoptosis accompanied by an upregulation of p53 have been noted in lung epithelial cells, and the Fas-FasL pathway was found to be upregulated in lung tissues from patients with IIPs [31, 32].

These results suggest that oxidative stress may participate in epithelial cell damage in patients with idiopathic interstitial pneumonias and that increased mitochondrial mass in lung epithelial cells may associate with increased oxidative stress.

Acknowledgements. The English used in this manuscript was revised by K. Miller (Royal English Language Centre, Fukuoka, Japan).

\section{References}

1. Kasai H. Analysis of a form of oxidative DNA damage, 8-hydroxy-2' deoxyguanosine, as a marker of cellular oxidative stress during carcinogenesis. Mutat Res 1997; 387: 147-163.

2. Maki H, Sekiguchi M. Protein MutT protein specifically hydrolyses a potent mutagenic substrate for DNA synthesis. Nature 1992; 355: 273-275.

3. Floyd RA. The role of 8-hydroxyguanine in carcinogenesis. Carcinogenesis 1990; 11: 1447-1450.

4. Ames BN, Shigenaga MK, Hagen TM. Oxidants, antioxidants, and the degenerative diseases of aging. Proc Natl Acad Sci USA 1993; 90: 7915-7922.

5. Kang D, Nishida J, Iyama A, et al. Intracellular localization of 8-oxo-dGTPase in human cells, with special reference to the role of the enzyme in mitochondria. J Biol Chem 1995; 270: 14659-14665.

6. Furuichi M, Yoshida MC, Oda $\mathrm{H}$, et al. Genomic structure and chromosome location of the human mutT homologue gene MTH1 encoding 8-oxodGTPase for prevention of A:T to $\mathrm{C}: \mathrm{G}$ transversion. Genomics 1994; 24: 485-490.

7. Kennedy $\mathrm{CH}$, Cueto R, Belinsky SA, Lechner JF, Pryor WA. Overexpression of hMTH1 mRNA: a molecular marker of oxidative stress in lung cancer cells. FEBS Lett 1998; 429: 17-20.

8. Tsutsui H, Ide T, Shiomi T, et al. 8-oxo-dGTPase, which prevents oxidative stress-induced DNA damage, increases in the mitochondria from failing hearts. Circulation 2001; 104: 2883-2885.
9. Gaston B, Drazen JM, Loscalzo J, Stamler JS. The biology of nitrogen oxides in the airways. Am J Respir Crit Care Med 1994; 149: 538-551.

10. Shi X, Ding M, Chen F, et al. Reactive oxygen species and molecular mechanism of silica-induced lung injury. J Environ Pathol Toxicol Oncol 2001; 20: Suppl. 1, 85-93.

11. Cantin AM, North SL, Fells GA, Hubbard RC, Crystal RG. Oxidant-mediated epithelial cell injury in idiopathic pulmonary fibrosis. J Clin Invest 1987; 79 : $1665-1673$.

12. Saleh D, Barnes PJ, Giaid A. Increased production of the potent oxidant peroxynitrite in the lungs of patients with idiopathic pulmonary fibrosis. Am J Respir Crit Care Med 1997; 155: 1763-1769.

13. Montuschi P, Ciabattoni G, Paredi P, et al. 8-Isoprostane as a biomarker of oxidative stress in interstitial lung diseases. Am J Respir Crit Care Med 1998; 158: 1524-1527.

14. Rahman I, Skwarska E, Henry M, et al. Systemic and pulmonary oxidative stress in idiopathic pulmonary fibrosis. Free Radic Biol Med 1999; 27: 60-68.

15. Jack CI, Jackson MJ, Johnston ID, Hind CR. Serum indicators of free radical activity in idiopathic pulmonary fibrosis. Am J Respir Crit Care Med 1996; 153: 1918-1923.

16. Richter C. Reactive oxygen and DNA damage in mitochondria. Mutat Res 1992; 275: 249-255.

17. American Thoracic Society. Idiopathic pulmonary fibrosis: diagnosis and treatment. International consensus statement. Am J Respir Crit Care Med 2000; 161: 646-664.

18. Kazerooni EA, Martinez FJ, Flint A, et al. Thinsection CT obtained at $10-\mathrm{mm}$ increments versus limited three-level thin-section CT for idiopathic pulmonary fibrosis: correlation with pathologic scoring. AJR 1997; 169: 977-983.

19. Ashcroft T, Simpson JM, Timbrell V. Simple method of estimating severity of pulmonary fibrosis on a numerical scale. J Clin Pathol 1988; 41: 467-470.

20. de la Monte SM, Luong T, Neely TR, Robinson D, Wands JR. Mitochondrial DNA damage as a mechanism of cell loss in Alzheimer's disease. Lab Invest 2000; 80: $1323-1335$.

21. Toyokuni S, Tanaka T, Hattori Y, et al. Quantitative immunohistochemical determination of 8-hydroxy-2'deoxyguanosine by a monoclonal antibody N45.1: its application to ferric nitrilotriacetate-induced renal carcinogenesis model. Lab Invest 1997; 76: 365374.

22. Asami S, Hirano $\mathrm{T}$, Yamaguchi R, Tomioka $\mathrm{Y}$, Itoh $\mathrm{H}$, Kasai $\mathrm{H}$. Increase of a type of oxidative DNA damage, 8-hydroxyguanine, and its repair activity in human leukocytes by cigarette smoking. Cancer Res 1996; 56: 2546-2549.

23. Inoue $\mathrm{M}$, Osaki $\mathrm{T}$, Noguchi $\mathrm{M}$, Hirohashi $\mathrm{S}$, Yasumoto K, Kasai H. Lung cancer patients have increased 8-hydroxydeoxyguanosine levels in peripheral lung tissue DNA. Jpn J Cancer Res 1998; 89: 691-695.

24. Collins A, Cadet J, Epe B, Gedik C. Problems in the measurement of 8-oxoguanine in human DNA. Report of a workshop, DNA oxidation, held in Aberdeen, UK, 19-21 January, 1997. Carcinogenesis 1997; 18: 1833-1836.

25. Cross CE, van der Vliet A, $\mathrm{O}^{\prime}$ Neill CA, Louie S, Halliwell B. Oxidants, antioxidants, and respiratory 
tract lining fluids. Environ Health Perspect 1994; 102 : Suppl. 10, 185-191.

26. Suhara T, Fukuo K, Sugimoto T, et al. Hydrogen peroxide induces up-regulation of Fas in human endothelial cells. J Immunol 1998; 160: 4042-4047.

27. Stridh H, Kimland M, Jones DP, Orrenius S, Hampton MB. Cytochrome c release and caspase activation in hydrogen peroxide- and tributyltininduced apoptosis. FEBS Lett 1998; 429: 351-355.

28. Uberti D, Yavin E, Gil S, Ayasola KR, Goldfinger N, Rotter V. Hydrogen peroxide induces nuclear translocation of p53 and apoptosis in cells of oligodendroglia origin. Brain Res Mol Brain Res 1999; 65: 167-175.

29. Miyashita T, Reed JC. Tumor suppressor p53 is a direct transcriptional activator of the human bax gene. Cell 1995; 80: 293-299.

30. Muller M, Wilder S, Bannasch D, et al. p53 activates the CD95 (APO-1/Fas) gene in response to DNA damage by anticancer drugs. J Exp Med 1998; 188: 2033-2045.

31. Kuwano K, Miyazaki H, Hagimoto $\mathrm{N}$, et al. The involvement of Fas-Fas ligand pathway in fibrosing lung diseases. Am J Respir Cell Mol Biol 1999; 20: 53-60.

32. Kuwano $\mathrm{K}$, Kunitake $\mathrm{R}$, Kawasaki $\mathrm{M}$, et al. P21Waf1/Cip1/Sdi1 and p53 expression in association with DNA strand breaks in idiopathic pulmonary fibrosis. Am J Respir Crit Care Med 1996; 154: 477483. 